\title{
PAPR Reduction of Wavelet-OFDM Systems Using Pilot Symbols
}

\author{
Kelvin Anoh ${ }^{\dagger}$, Augustine Ikpehai ${ }^{\dagger}$, Khaled Rabie $^{\dagger}$, Bamidele Adebisi ${ }^{\dagger}$ and Wasiu Popoola ${ }^{\ddagger}$ \\ ${ }^{\dagger}$ School of Engineering, Manchester Metropolitan University, M1 5GD UK \\ ${ }^{\ddagger}$ Institute for Digital Communications, School of Engineering, University of Edinburgh, Edinburgh EH9 3JL, UK \\ ${ }^{\dagger}\left[\right.$ k.anoh, a.ikpehai, k.rabie, b.adebisi]@mmu.ac.uk, ${ }^{\ddagger}$ w.popoola@ed.ac.uk
}

\begin{abstract}
Wavelet transform is at the heart of IEEE 1901 power line communication standard which uses orthogonal frequency-division multiplexing (OFDM) scheme. Examples of wavelets used in designing wavelet-based OFDM (WOFDM) have been shown as possessing lower peak-to-average power ratio (PAPR) than the conventional fast Fourier transform (FFT)-based OFDM. In this study, we investigate 29 different orthogonal wavelets that can be used in designing WOFDM in terms of PAPR and sidelobes behaviour of the filters that enable these wavelets. Our WOFDM model shows that most orthogonal wavelets with increasing filter coefficient lengths achieve better PAPR. In addition, we also demonstrate a method of pilot-assisted OFDM (PA-OFDM) processing to further reduce the PAPR of the WOFDM systems. This technique achieves PAPR reduction with no added complexity and no extra bandwidth cost.
\end{abstract}

Index Terms-Wavelets, OFDM, PAPR, pilot assisted OFDM, power line communication, sidelobes

\section{INTRODUCTION}

Although there are different candidate waveforms for emerging modern communication system standards (e.g. 5G, PLC, DSRC, optical-fibre communication, etc.) [1]-[5], data multiplexing is still a burgeoning challenge in maximizing spectrum utilization. Conventionally, orthogonal frequencydivision multiplexing (OFDM) is used to multiplex transmit data due to its efficiency in maximizing a given spectrum and robustness over multipath fading. OFDM finds application in power line communication (PLC) systems [6]. By using discrete Fourier transform which can efficiently process signals using the fast Fourier transform (FFT) algorithm, OFDM divides wide bandwidths into $N$ smaller orthogonal channels thus increasing the symbol time. However, it requires up to $1 / 4$ of the bandwidth to successfully combat the intersymbol interference (ISI) arising from delay spread of the channel by using cyclic prefixing $(\mathrm{CP})$ of length $L_{c p}$; this wastes as much spectrum. This problem expands the processing time and expends the system power by $L_{c p} /\left(N+L_{c p}\right)$ and also dissipates very high sub-band frequency energy response (sidelobes) [7] increasing adjacent channel interferences. These problems can be overcome in the design of multicarrier systems by using a different modulating kernel than FFT, namely wavelets [7], [8]. Compared to FFT-OFDM, wavelet-based OFDM (WOFDM) has economical bandwidth management and offers better (smaller) spectral leakage [9], [10].

Wavelets are therefore alternative design kernel for processing multicarrier data signals. The problem with the type of wavelets described in [7], [8] is that the data symbols must be real, thus the peak-to-average power ratio (PAPR) will be surely higher than that of the conventional OFDM systems [8], [11]. Beyond this, in the literature, there are many different kernels and filters [12]-[18] that implement wavelets whose input signals may not be restricted to real data symbols only. Such wavelets can dispense with better PAPR performance than FFT-OFDM (e.g. [19]). These have not been explored comprehensively, for example in terms of PAPR reduction capabilities. Different wavelet families [18] can be implemented in terms of discrete wavelet transform (DWT) or wavelet packet transform (WPT) [20]. We will adopt the WPT approach which achieves full realization of subcarriers with minimum decomposition levels unlike the DWT approach.

A common problem in the design of OFDM systems is that the PAPR is usually high due to the coherent addition of energies from the adjacent sub-bands [11]. In the literature, there are different techniques that have been proposed to address the PAPR problem which could be applied before or after OFDM modulation [21]-[23]. While companding [24], [25] and clipping [26] are post-modulation PAPR reduction schemes, they destroy the orthogonality of the subcarriers. On the other hand, using partial transmit sequence and selective mapping (SLM) [8] increases the complexity of the system, and over fading channel requires that pilots be added to estimate the channel. These pilots have now been shown to enhance PAPR reduction [11], [27] and do not require sending side information to the receiver which increases system complexity [27]. That will be adopted in this study.

The ideal of pilot-assisted OFDM (PA-OFDM) in reducing PAPR achieves reduced system complexity. In this study, we adopt the use of pilots, which are widely known to enhance channel estimation and reduce bit error probability, to also reduce the PAPR as in [11], [28], [29] for 29 different orthogonal wavelets. Wavelets are alternative multicarrier modulation kernels used in the design of OFDM-like multicarrier systems that exist in different forms [10], [13], [15], [17]. It has the property of resolving frequencies in blocks of time of interest just like the short-time Fourier transform.

In the literature, it has been shown that orthogonal wavelets are more suitable for multicarrier signal transmission over fading channels due to the orthogonality of the basis functions [18]. Also, it has also been shown that some wavelets have lower PAPR than FFT-based OFDM systems [19]. This 
however not the case for all wavelets as different filters process OFDM signals with different capabilities. Thus, we show the PAPR performances of 29 different orthogonal wavelets and also reduce the conventional PAPR limits of these wavelets by using pilot signals, which can as well be used for channel estimation. This study also reveals that the PAPR performances of the WOFDM systems are dependent on the filter that enables the wavelet.

\section{System MODEL}

This study considers an OFDM model design using wavelets as the modulating kernel. It also involves pilots conventionally applied for channel estimation [28], but in this case used for PAPR reduction by rotating the phase of each OFDM signal as in [11]. The approach reduces the complexity of additional PAPR reduction constraints on the system, unlike deliberate clipping technique [26] which incurs $2 r+1$ IFFT/FFT complexity; where $r$ is the number of clipping and filtering iterations. The PA-OFDM approach does not involve amplitude distortion like companding [21], [25] which generates in-band noise that diminish the received signal integrity.

Consider some quadrature amplitude modulated (QAM) data symbols $\mathrm{D}_{\bar{n}} \in \mathbb{C}^{(N-P) \times 1}$. The pilot-assisted PAPR reduction in OFDM system may be thought of as being realized from typical SLM PAPR reduction process involving coding of data symbols, using a mask say $\mathbf{M}_{i}^{u}=A_{i, u} \exp \left(j \theta_{i, u}\right)$ as in [29], where $\forall i=1, \cdots, P$ represents the number of pilots and $u=1, \cdots, U$ represents the number of pilot sequence. In this case, the PA-OFDM data can be represented as

$$
\overline{\mathrm{X}}_{\bar{n}}=\mathrm{D}_{\bar{n}} \otimes \mathrm{M}_{i}^{u} \quad \forall i \in P, \bar{n} \in N-P
$$

where $\overline{\mathrm{X}}_{\bar{n}} \in \mathbb{C}^{(N-P) \times 1} \forall \bar{n}=0,1, \cdots, N-P$ represent the conventional frequency-domain content of the OFDM symbol. It follows that the phase of each data symbol is rotated by $\theta_{i, u}$ of the pilot and the amplitude scaled by $A_{i, u}$. Afterwards, we insert the pilot symbols so that $\mathrm{X}_{m}=\overline{\mathrm{X}}_{\bar{n}}+\overline{\mathrm{X}}_{p, m}^{u}$ $\forall u=1, \cdots, U$ becomes the frequency domain-content OFDM symbol with pilots (see [29]). This can be explicitly represented as

$$
\begin{aligned}
& \mathbf{X}_{m}=\operatorname{Diag}\left(\mathbf{I}_{N}\left[\begin{array}{c}
\overline{\mathbf{X}}_{\bar{n}} \\
\mathbf{1}_{P \times 1}
\end{array}\right]\right)\left[\begin{array}{c}
\mathbf{1}_{(N-P) \times 1}-\overline{\mathbf{X}}_{p, m}^{u}
\end{array}\right] \\
& \forall m=0,1, \cdots, N-1
\end{aligned}
$$

where $\overline{\mathrm{X}}_{p, m}^{u} \in \mathbb{C}^{P \times 1}$ represent the pilot symbols. In this study, $\theta_{i, u}$ is chosen from 0 and $\pi$ [11]. To ensure that all peaks are measured, $\mathrm{X}_{m}$ is usually oversampled by a factor $\ell$ which subtends an oversized FFT of $\ell N$. The resulting symbol is then transformed into time-domain by the inverse Fourier transform operator thus

$$
\begin{aligned}
& \mathbf{x}_{n}=\mathbf{F}^{\prime}\left(\left[\begin{array}{ll}
\mathrm{X}_{m} & \mathbf{0}_{(\ell N-N) \times 1}
\end{array}\right]\right) \\
& =\mathbf{F}^{\prime}\left(\left[\overline{\mathbf{X}}_{\bar{n}}+\overline{\mathbf{X}}_{p, m}^{u} \mathbf{0}_{(\ell N-N) \times 1}\right]\right) \\
& \mathbf{F}^{\prime}=\frac{1}{\sqrt{N}}\left(\mathbf{I}_{N} e^{j 2 \pi \frac{n k}{N}}\right) \forall k, n=0,1, \cdots, \ell N-1
\end{aligned}
$$

where $\mathbf{I}_{N}$ is an identity matrix and $1 / \sqrt{N}$ is the normalization factor; the essence of this normalization is to ensure that the energy of the information symbol does not change. Unlike the conventional pilot OFDM system, the above form of SLM by using PA-OFDM adds the flavour of processing OFDM symbol frames with varying pilot tones. Afterwards, the OFDM symbol with the minimum PAPR is transmitted.

From (1), the $\mathrm{D}_{n} \otimes \mathrm{M}_{i}^{u}$ operation designates that the masking operation of the pilot data unto the OFDM symbol data follow a linear operation. Although the data symbols form the wellknown multicarrier symbol of the OFDM kind, the kernel that enables the system is subtended by the wavelet processing kernel which we shall discuss in the following section.

\section{A. Wavelet OFDM Signal Processing}

A WOFDM system can be designed by using any suitable orthogonal mother wavelet to process input signals. Among these orthogonal wavelets, there is so far no unified theory to the performance optimality of the myriad of available wavelets when applied to OFDM systems. Thus we investigate the performance using extensive computer simulation. It is common to state that wavelets are enabled through filter banks consisting of low-pass filter (LPF) and high-pass filter (HPF). The LPF is related to the scaling function by the dilation equation

$$
\varphi(l)=\sqrt{2} \sum_{\tau=0}^{L-1} h[\tau] \varphi(2 l-\tau) .
$$

On the other hand, the wavelet function can as well be realized from weighting the scaling using an HPF, $g$, such as

$$
\psi(l)=\sqrt{2} \sum_{\tau=0}^{L-1} g[\tau] \varphi(2 l-\tau)
$$

where the parameter $\sqrt{2}$ is a normalization parameter, although $\int_{-\infty}^{\infty} \varphi(x) d x=1$. Both the LPF and HPF are related to each other as $g_{\tau}=(-1)^{\tau} h_{L-\tau-1}, \forall \tau=0, \cdots, L-1$; these are the so-called filter bank components. The $\varphi(n)$ and $\psi(n)$ parts of the signal give rise to approximate and detail coefficients of the signal being processed. It follows that when given a signal $x_{k}, k=0,1, \cdots, \ell N-1$ as the sample coefficients of $x$, then we can discuss the detail $(d)$ and the approximate $(a)$ parts of the symbol as follows

$$
\begin{gathered}
a^{j}[n]=\sum_{n=0}^{L-1} h[n] x^{j-1}[n+2 \tau] \\
d^{j}[n]=\sum_{n=0}^{L-1} g[n] x^{j-1}[n+2 \tau+1]
\end{gathered}
$$

These exist within the scales of $j \geq 1$ and periods of $2^{n-j}$ [30]; most times, the term $j$ is usually referred to as decomposition level. The detail and approximate parts of the symbol in (6) can be summarized as follows

$$
s[n]=\sum_{k=0}^{\ell N-1} a[k]+\sum_{k=0}^{\ell N-1} d[k] \forall n=0,1, \cdots, \ell N-1
$$

usually described as wavelet packets which are enabled by different mother wavelets. We shall be using (7) henceforth as our time-domain processed signals. 


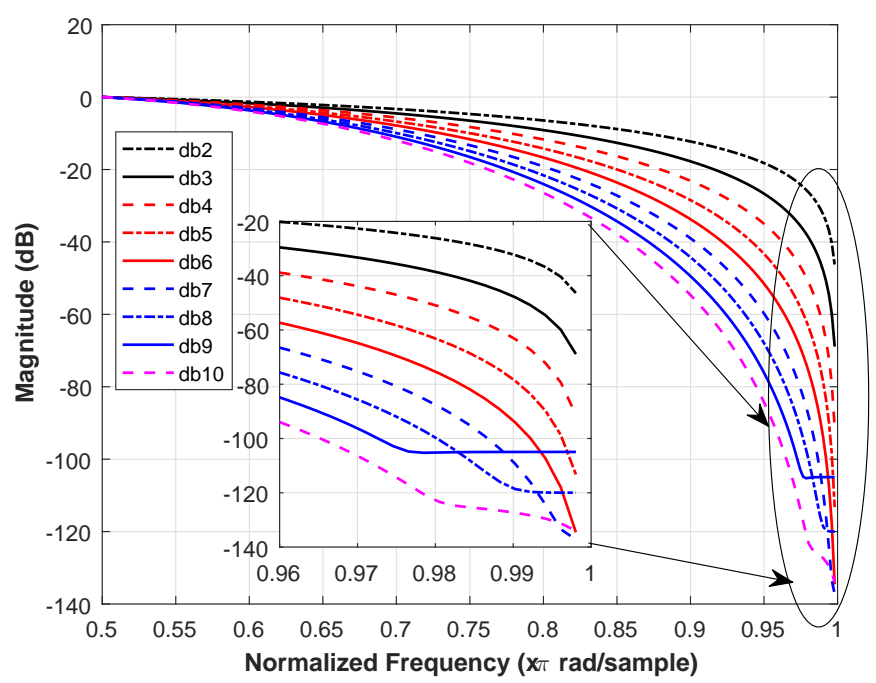

Figure 1. Performance comparison of side-lobes of Daubechies wavelets (db2 $-\mathrm{db} 10)$

\section{B. Pilot-Assisted OFDM PAPR Reduction Scheme}

Recall in (1) that our interest is in using pilot tones to reduce the PAPR of OFDM symbols - the OFDM symbol here is enabled using wavelet transform. Explicitly, the transmitted OFDM signal follows from that which satisfies the following optimization problem [11], namely

$$
\begin{aligned}
s_{p, t}^{u}[n] & =\min _{1 \leq t \leq T}\left(P A P R_{p, t}^{u}\right) \quad \forall t=1,2, \cdots, T \\
u & =1, \cdots, U \\
& =1, \cdots, P .
\end{aligned}
$$

where the PAPR is measured as follows

$$
\operatorname{PAPR}_{p, t}^{u}=\frac{\max _{n=0,1, \cdots, N-1}\left(\left|s_{p, t}^{u}[n]\right|^{2}\right)}{\mathbb{E}\left\{\left|s_{p, t}^{u}[n]\right|^{2}\right\}} \forall t=1,2, \cdots, T .
$$

In terms of complementary cumulative density function (CCDF), the PAPR is discussed as

$$
\begin{aligned}
\mathrm{CCDF} & =\operatorname{Pr}\left\{P A P R_{p, t}^{u}>\mathrm{PAPR}_{0}\right\} \\
& =\left[1-\left(1-\exp \left(-\mathrm{PAPR}_{0}\right)\right)^{\ell N}\right] .
\end{aligned}
$$

Here, $\operatorname{Pr}\{\cdot\}$ represents the probability of $\{\cdot\}$ and $\operatorname{PAPR}_{0}$ is a reference PAPR threshold. We remark that $s_{p, t}^{u}[n]$ satisfies the minimum PAPR metric among all the OFDM signal frames of length $\mathbb{C}^{\ell N \times 1}$ after $1 \leq t \leq T^{t h}$ iterations.

\section{Sidelobe performance of different Mother wavelets}

Wavelets from the orthogonal family have distortionless sidelobes [12]-[14]. We evaluate the performances of different wavelets in terms of the side-lobe performances which has the potential to influence the energy of the adjacent subbands. All wavelets are enabled by filters and these filters subtend the mother wavelets. The structure of the mother wavelets and the filters influence how they process OFDM

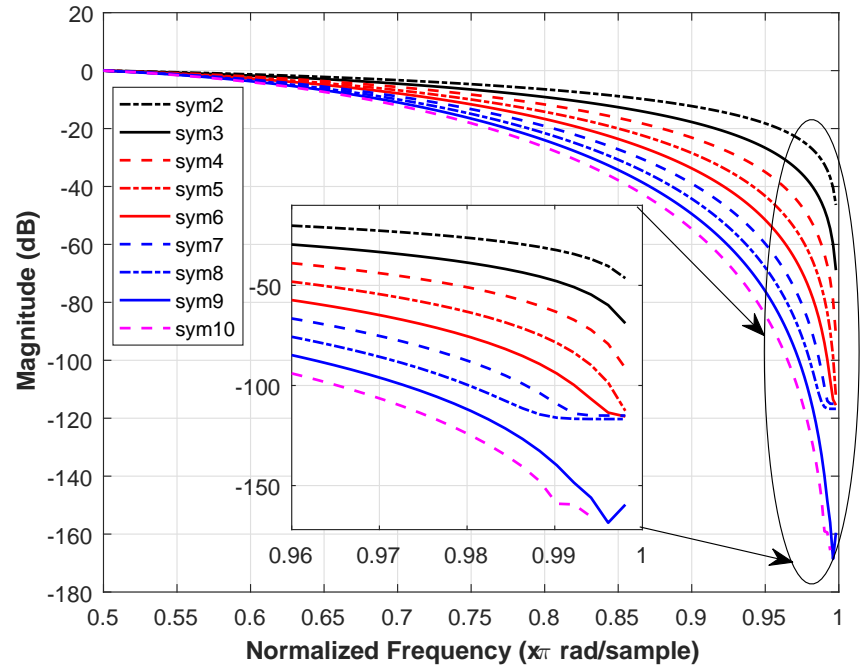

Figure 2. Performance comparison of first 9 symlet wavelets in terms of sidelobes

signals. We do not consider Haar wavelet (or db1) as they have only two filter coefficients. Most times db1 is usually not considered as a true-wavelet because it is not continuous and thus not differentiable. In this study, we consider the most popular wavelets namely Daubechies (db), Coiflets (coif), Symlets (sym) and Fejer-Korovkin (fk) respectively. $\mathrm{db} \nu$ or $\operatorname{sym} \nu$ implies that wavelet "db" or "sym" has $2 \times \nu$ filter coefficients. Unlike the Daubechies and the Symlets wavelets, coiflet wavelets follow a different convention. For example there are coiflet 1 - coiflet 5 , growing by 6 filter coefficients; e.g., there are 6 coefficients in coif1, 12 coefficients in coif2, 18 coefficients in coif 3 , and so on.

In [7], it was shown that wavelets have better suppressed sidelobes than conventional FFT-OFDM systems. That study did not explore other well-known wavelets, such as the orthogonal wavelets described in the literature [12], [14], [17], [20]. In the following, we demonstrate the filter response of these wavelets showing the performance of the sidelobes in Figs. 1-4. The sidelobes of all the wavelets are distortionless. The like likelihood of increasing the energy in the adjacent subband is thus eliminated. However, some wavelets have better suppressed sidelobes than others. For example, we find that as the filter length increases, the sidelobes reduce which will influence the likelihood of increasing the energy in adjacent sub-bands; this is valid for all the different wavelet families. The phenomenon is related to the increasing filter coefficients offering better resolution to the input signal. Specifically, $\mathrm{db}$ wavelets achieve about $-140 \mathrm{~dB}$ suppression, while Symlets achieve about $-170 \mathrm{~dB}$ response. Coiflets achieve about $120 \mathrm{~dB}$ while fk-wavelets achieve about $-50 \mathrm{~dB}$ suppression. Notice that fk-wavelets have quite fractal sidelobe responses except fk4; this will affect the energy dissipation in the adjacent sub-bands and thus the PAPR performance.

Of special interest however, notice that the depth of the sidelobes of db, sym, coif and fk-wavelets are not the same. In other words, these wavelets with poor suppressed sidelobes 


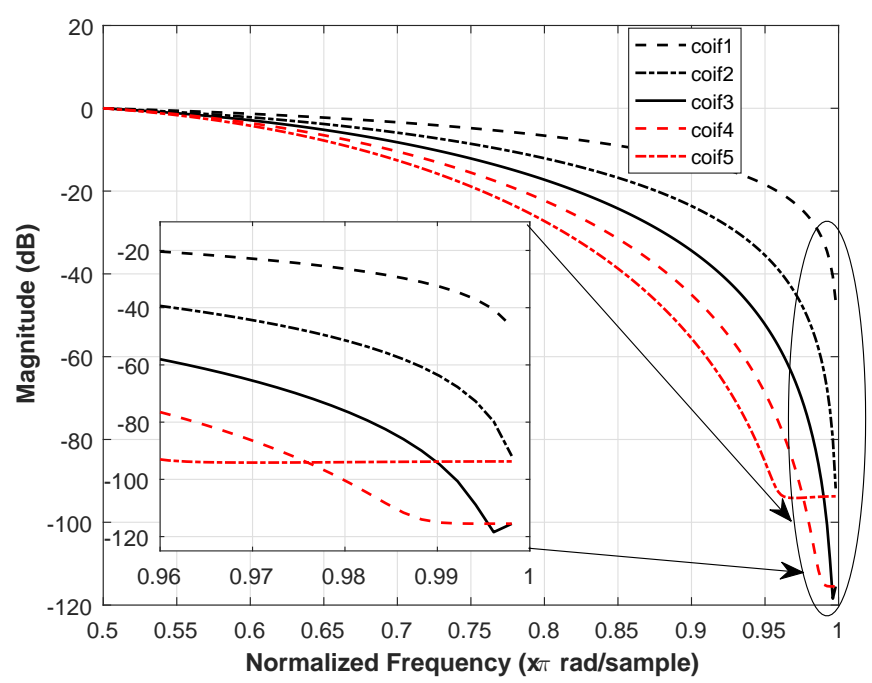

Figure 3. Performance comparison of all the 5 coiflet wavelets in terms of sidelobes of the filters that enable each wavelet

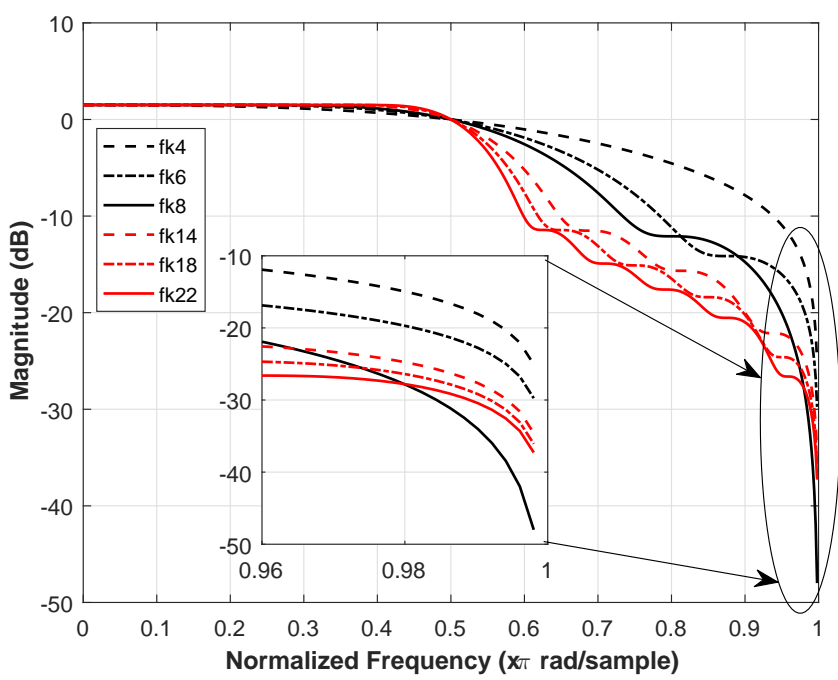

Figure 4. Performance comparison of all the Fejer-Korovkin wavelets in terms of the sidelobes of the filter responses

will create more adjacent band interference than others. However, among all these families, the wavelets from sym-family dispense with the lowest sidelobe magnitudes. It is known that some wavelets have worse PAPR performance than the FFTbased OFDM [8]. That study, will be here, corroborated as some orthogonal wavelets have PAPR performances slightly worse than that of the conventional FFT-OFDM (see the next section).

\section{RESULTS AND DISCUSSION}

The system model involves the conventional OFDM-like system architecture in which some $N-P$ random data symbols are generated $(N=64)$, where $n=0,1, \cdots, N-1$ designates the total number of multicarrier sub-bands over which the data symbols are multiplexed and $P=1$ represents the number of pilots used. These data symbols are then modulated using the

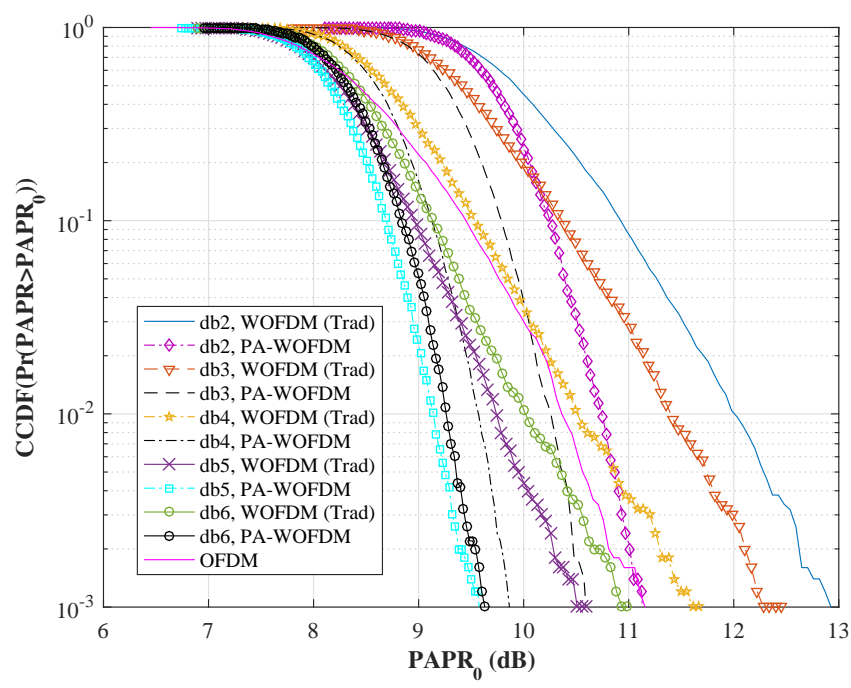

Figure 5. Comparison of PAPR performances of different Daubechies wavelets (involving db2-6), notice that Haar (db1) is not presented due to the reason stated earlier. These performances are based on both the PA-OFDM design and the conventional OFDM architecture with wavelet transform as the modulating kernel. All wavelet transforming kernels presented are from the orthogonal wavelet family.

16-QAM and later transformed using different wavelet filters. We normalized the output data symbols to ensure that the average energy follows $\sigma_{s}^{2}=\frac{1}{2} \mathbb{E}\{s[n]\}=1$. Then, to avoid missing some peaks of the symbols, we oversample the data symbols $\ell=4$ times, so that an $\ell N$ oversized subcarriers are required. The resulting data symbols are then linearly phaserotated using the phase of the pilot symbols locked to 0 and $\pi$. Afterwards, we measure the PAPR of the wavelet-OFDM symbols and the results are presented in the following figure. This process is repeated $T=6$ times and averaged over $5 \times 10^{3}$ simulation runs, each time selecting the minimum PAPR achieved WOFDM symbol. Throughout the study, we set $A_{i, u}=1, \forall i=1, \cdots, P$ and $\forall u=1, \cdots, U$, where $U=1$.

The PAPR performances of the different orthogonal wavelets are shown in Fig. 5. We compare the performances of each wavelet filter kernel as an OFDM modulating transform with own-self when processed with and without pilot assistance. The comparison also involves the performances of different wavelets with one another in terms of their respective PAPR. Clearly, db2 wavelet shows the worst PAPR performance among the all other wavelets. Although it has the smallest length of filters (4-filters coefficients), which may gain slight processing time, its PAPR performance is not as good as others. However, when operated as PA-OFDM, it can be seen that the PAPR performance improves by $3 \mathrm{~dB}$ gain at $10^{-3}$ CCDF. In Fig. 6, we find that the PAPR is reduced as the filter length increases with db5 performing better than the other wavelets (db7-db9).

From Fig. 7, one finds that all the wavelets perform differently. However, the wavelet with longest filter coefficients (sym6) among (sym2 -sym6) achieves the lowest PAPR reduction. This performance further improves as the filter lengths 


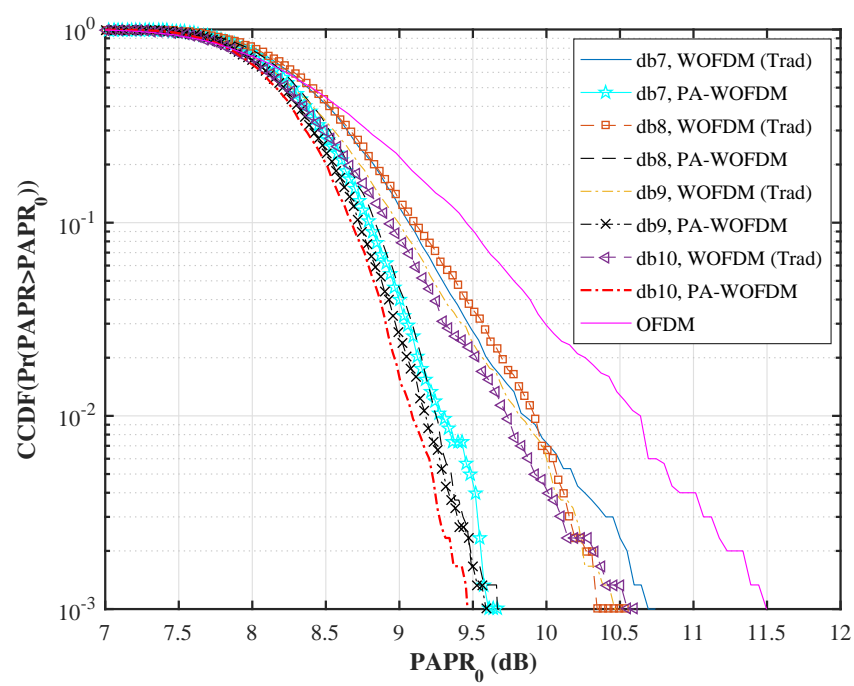

Figure 6. Comparison of PAPR performances of different orthogonal wavelets (db7 - db10) wavelet filters based on PA-OFDM design and the conventional OFDM.

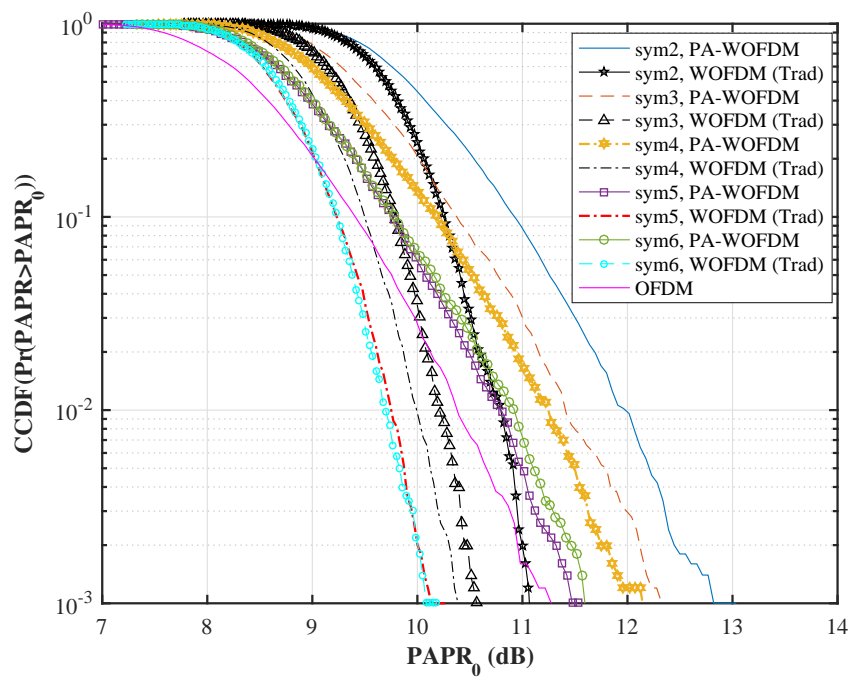

Figure 7. PAPR performance evaluation of WOFDM of the first 5 Symlet wavelets (sym2 - sym6), including when modulated using pilot-symbol assistance

increase as shown in Fig. 8 and it is observed that sym7 achieves the lowest PAPR reduction.

The performance trend as described above continues for coiflets as shown in Fig. 9. While operated the WOFDM with pilots reduces the PAPR by up to $1.5 \mathrm{~dB}$ for coiflets, the most performing is coif5 corresponding to the one with the longest filter coefficients.

Lastly, we examine in Fig. 10 the PAPR performances for fk-wavelets. Results show that the wavelet with the longest filter coefficients among the "fk" wavelets (namely fk22) achieves the most PAPR reduction performance. However, the degree of PAPR reduction achieved is about $1 \mathrm{~dB}$.

Among all the wavelets considered, using wavelets with higher order filter length achieve the better PAPR reduction

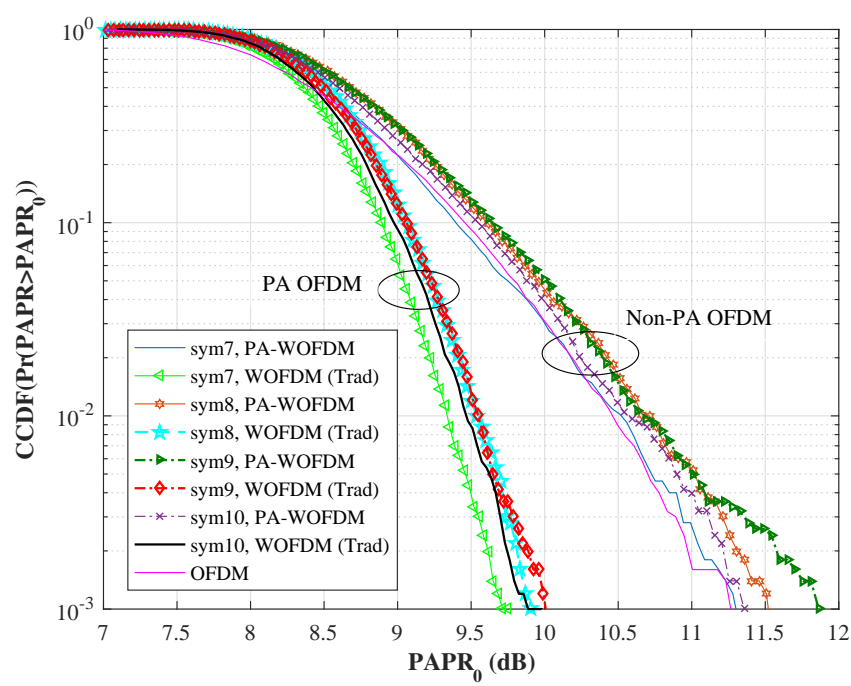

Figure 8. PAPR performance evaluation of Symlet (sym7 - sym10) wavelets as an OFDM kernel, including when modulated using some pilot-symbol assistance

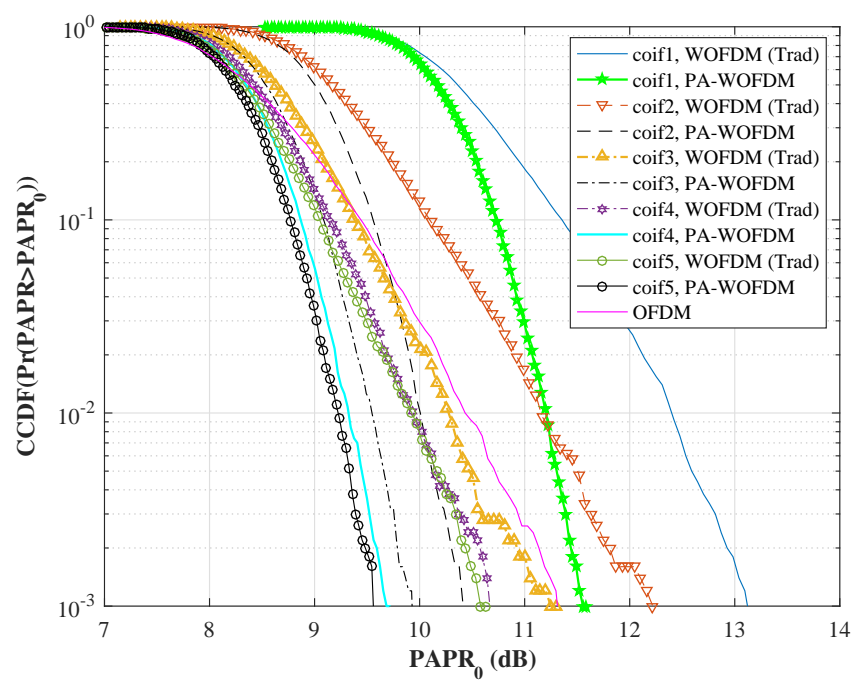

Figure 9. Performance evaluation of PAPR of WOFDM for different wavelets, including when modulated using some pilot-symbol assistance (coif1, coif2, coif3, coif4, coif5)

when operated with and without using PA-OFDM. We infer that different wavelets performs differently and that most wavelets with increasing filter length achieve better PAPR; this advantage is achieved at the cost of processing time. Recall that we stated that PAPR reduction can be achieved before or after OFDM modulation. Thus, the PAPR performances achieved via pilot assistance can then be enhanced using clipping or companding after the OFDM modulation.

\section{CONCLUSION}

In this study, we have enumerated a number of different merits that wavelets provide as an alternative multicarrier design kernel to FFT in the design of OFDM systems. OFDM scheme is used to enable power line communication systems. 


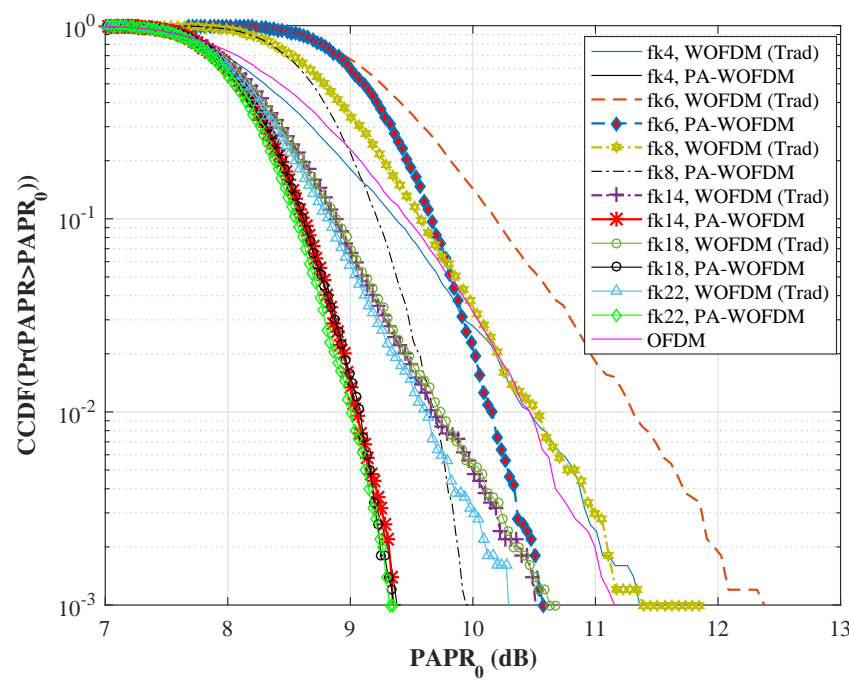

Figure 10. Performance evaluation of different wavelets, including when modulated using some pilot-symbol assistance (fk4, fk6, fk8, fk14, fk18, fk22)

In addition to providing well suppressed side-lobes which will result in substantially reduced interference during communication, wavelets were shown to offer decent PAPR measures. Although it has been controversially advertised in the literature that wavelets outperform FFT-based OFDM in terms of PAPR, it is shown in this study that not all wavelets have this property. We demonstrated these using 29 different orthogonal wavelets in terms of PAPR reduction for multicarrier systems. It is shown that the PAPR performances of these orthogonal wavelets yield more to increasing filter lengths. To improve the PAPR, we rotated the phase of the OFDM symbols using that of pilot symbols (which are usually used in channel estimation over fading channels) to reduce the PAPR. This shows that the technique provides great advantage without added complexity like other PAPR reduction schemes such as clipping. Finally, we showed that the side-lobe suppression of wavelet sub-band filters reduce with increasing filter length. This reduces the coherent additions in the energy of the adjacent sub-bands leading to better PAPR for Daubechies and Symlet wavelets.

\section{ACKNOWLEDGEMENT}

This research has been carried out within the "CityVerve: IoTs and Smart Cities Demonstrator" project funded by Innovate UK (Innovate UK with project reference: 102561).'

\section{REFERENCES}

[1] E. Başar, Ü. Aygölü, E. Panayırcı, and H. V. Poor. Orthogonal frequency division multiplexing with index modulation. IEEE Trans. Signal Process., 61(22):5536-5549, Nov. 2013.

[2] R. Gerzaguet et al. The 5G candidate waveform race: a comparison of complexity and performance. EURASIP J. Wireless Commun. Netw. 2017(1):13, 2017.

[3] L. Lampe. Power Line Communications: Principles, Standards and Applications from Multimedia to Smart Grid. John Wiley \& Sons, 2016.

[4] M. Yousefi and X. Yangzhang. Linear and nonlinear frequency-division multiplexing. In Proc. 42nd European Conf. Opt. Commun., pages 1-3. VDE, 2016
[5] Y. J. Li. An overview of the dsrc/wave technology. In Int. Conf. Heterogeneous Netw. Quality, Reliability, Security and Robustness, pages 544-558. Springer, 2010.

[6] K. Anoh, B. Adebisi, K. M. Rabie, M. Hammoudeh, and H. Gacanin. On companding and optimization of OFDM signals for mitigating impulsive noise in power-line communication systems. IEEE Access, 5:2181821830, Sept. 2017.

[7] M. Hoch. Comparison of ConvOFDM and Wavelet-OFDM for NarrowBand Power Line Communications. Universität Erlangen-Nürnberg, Germany, 2010.

[8] M. Hoch, S. Heinrichs, and J. B. Huber. Peak-to-average power ratio and its reduction in wavelet-OFDM. In Int. OFDM Workshop, pages 56-60. Hamburg, 2011.

[9] S. Galli, H. Koga, and N. Kodama. Advanced signal processing for PLCs: Wavelet-OFDM. In IEE ISPLC 2008., pages 187-192, 2008.

[10] A. Farhang, M. M. Kakhki, and B. Farhang-Boroujeny. Wavelet-OFDM versus filtered-OFDM in power line communication systems. In 2010 5th Int. Symposium Telecom., pages 691-694, Dec 2010.

[11] W. O. Popoola, Z. Ghassemlooy, and B. G. Stewart. Pilot-assisted PAPR reduction technique for optical OFDM communication systems. J. Lightwave Technol., 32(7):1374-1382, 2014.

[12] K. Anoh, J. Noras, R. Abd-Alhameed, S. Jones, and K. Voudouris. A new approach for designing orthogonal wavelets for multicarrier applications. AEU-Int. J. Electron. Commun., 68(7):616-622, 2014.

[13] K. Anoh, R. Abd-Alhameed, O. Ochonogor, Y. Dama, S. Jones, and T. Mapoka. Performance evaluation of raised-cosine wavelet for multicarrier applications. IJEE, 1(3):1-5, 2014.

[14] K. Anoh, T. Mapoka, R. Abd-Alhameed, O. Ochonogor, and S. Jones. On the application of raised-cosine wavelets for multicarrier systems design. IRECAP, 4(4):143 - 150, 2014.

[15] D. E. Newland. Harmonic wavelet analysis. In Proc. Royal Society of London A: Math. Physical and Eng. Sci., volume 443, pages 203-225. The Royal Society, 1993.

[16] D. E. Newland. An introduction to random vibrations, spectral \& wavelet analysis. Courier Corporation, 2012.

[17] I. Daubechies. Ten lectures on wavelets. SIAM, 1992

[18] O. Anoh, R. Abd-Alhameed, S. Jones, J. Noras, Y. Dama, A. Altimimi, N. Ali, and M. Alkhambashi. Comparison of orthogonal and biorthogonal wavelets for multicarrier systems. In IEEE 8th IDT, pages 1-4, 2013.

[19] M. Gautier, C. Lereau, M. Arndt, and J. Lienard. PAPR analysis in wavelet packet modulation. In 3rd IEEE Int. Symp. Commun. Contr. Signal Process., pages 799-803, 2008.

[20] O. Anoh, N. Ali, R. Abd-Alhameed, S. Jones, and Y. Dama. On the performance of DWT and WPT modulation for multicarrier systems. In IEEE 17th Int. Workshop CAMAD, pages 348-352, 2012.

[21] K. Anoh, B. Adebisi, K. M. Rabie, and C. Tanriover. Root-Based Nonlinear Companding Technique for Reducing PAPR of Precoded OFDM Signals. IEEE Access, PP(99):1-1, 2017.

[22] K. Anoh, C. Tanriover, B. Adebisi, and M. Hammoudeh. A new Approach to Iterative Clipping and Filtering PAPR Reduction Scheme for OFDM Systems. IEEE Access, Sept. 2017.

[23] Y. Rahmatallah and S. Mohan. Peak-To-Average Power Ratio Reduction in OFDM Systems: A Survey And Taxonomy. IEEE Commun. Surveys Tuts., 15(4):1567-1592, Fourth Quarter 2013.

[24] X. Wang, T. T. Tjhung, and C. S. Ng. Reduction of peak-to-average power ratio of OFDM system using a companding technique. IEEE Trans. Broadcast., 45(3):303-307, Sept. 1999.

[25] B. Mohanty and S. Ramavath. A companding technique for PAPR reduction in DWT OFDM systems. In IEEE Int. Conf. Advanced Commun., Contr. Comput. Technol., pages 837-841, May 2014.

[26] J. Armstrong. Peak-to-average power reduction for OFDM by repeated clipping and frequency domain filtering. Electronics Letters, 38(5):246247, Feb. 2002.

[27] M. J. Fernandez-Getino Garcia, O. Edfors, and J. M. Paez-Borrallo. Peak power reduction for OFDM systems with orthogonal pilot sequences. IEEE Trans. Wireless Commun., 5(1):47-51, Jan. 2006.

[28] Z. Tang, R. Cannizzaro, G. Leus, and P. Banelli. Pilot-assisted timevarying channel estimation for OFDM systems. IEEE Trans. Signal Process., 55(5):2226-2238, May 2007.

[29] K. Wesołowski. On the PAPR minimization using selected mapping algorithm in pilot-assisted OFDM systems. In European Wireless Conf., 2007.

[30] G. Beylkin, R. Coifman, and V. Rokhlin. Wavelets in numerical analysis. Wavelets and their applications, 181, 1992. 